\title{
ANALYSIS AND APPLICATION OF MECHANICAL SYSTEM RELIABILITY MODEL BASED ON COPULA FUNCTION
}

\author{
Hai AN \\ Hang YIN \\ Fukai HE \\ School of Aerospace and Civil Engineering, Harbin Engineering University, Harbin 150001, \\ Heilongjiang, China
}

\begin{abstract}
There is complicated correlations in mechanical system. By using the advantages of copula function to solve the related issues, this paper proposes the mechanical system reliability model based on copula function. And makes a detailed research for the serial and parallel mechanical system model and gets their reliability function respectively. Finally, the application research is carried out for serial mechanical system reliability model to prove its validity by example. Using Copula theory to make mechanical system reliability modeling and its expectation, studying the distribution of the random variables (marginal distribution) of the mechanical product' life and associated structure of variables separately, can reduce the difficulty of multivariate probabilistic modeling and analysis to make the modeling and analysis process more clearly.
\end{abstract}

Keywords: copula function, mechanical system reliability, model

\section{INTRODUCTION}

There is complex correlations between each unit of the mechanical system and its failure models. There are a lot of analysis about the mechanical system reliability, but the studies to analyze the reliability from the correlation of mechanical system are few. In recent years, some scholars apply the connection function copula to discuss the correlation in financial, insurance and engineering, and achieve satisfactory results [1-3]. Literature [4-5] discussed some studies that the copula function is used in mechanical system reliability, but there were no deep study. Anyway, they contributed the reference value for the Copula function' deep study in terms of the mechanical system reliability. Based on the existing theories, this paper constructs the mechanical system reliability model based on copula function, and apply it to practice.

\section{THEORETICAL BASIS}

\section{DEFINITION AND NATURE OF COPULA FUNCTION}

In 1959, Sklar decomposed a joint distribution into $\mathrm{k}$ edge distributions and a Copula function, and this Copula function can describe the correlation among variables[6]. So, actually, Copula function is a function that connects the joint distribution of a multivariate random variables with their respective marginal distribution[7]. Use $R^{\mathrm{n}}$ to note the expand n-dimensional space (n is 
any positive integer), $\mathrm{a}=\left(a_{1}, a_{2}, \cdots, a_{n}\right)$ represents the point in $R^{\mathrm{n}}$, for all the $\mathrm{k}$, if $a_{k} \leq b_{k}$, then $a \leq b$. If $a \leq b$ use $\quad[\mathrm{a}, b]=\left[a_{1}, b_{1}\right] \times\left[a_{2}, b_{2}\right] \times \cdots \times\left[a_{n}, b_{n}\right] \quad$ represents n-dimensional cube, and its volume is noted $V_{c}([a, b])$. $\mathrm{n}$-Copula function has the following basic properties:

(1) $C\left(\mathrm{u}_{1}, u_{2}, \cdots, u_{n}\right)$ is the $\mathrm{n}$-distribution function which is defined in $[0,1]^{n}$, that is C: $[0,1]^{n} \rightarrow[0,1]$;

(2) For any variable $\mathrm{u}_{i}, i=1,2, \cdots, n, C\left(\mathrm{u}_{1}, u_{2}, \cdots, u_{n}\right)$ is monotonically increasing all the time;

(3) $C_{\mathrm{i}}$, the marginal distribution of $C\left(\mathrm{u}_{1}, u_{2}, \cdots, u_{n}\right)$ meets $\quad C_{i}\left(u_{i}\right)=C\left(1, \cdots, 1, u_{i}, 1, \cdots, 1\right)=u_{i}, \quad$ in $\quad$ which, $u_{i} \in[0,1], i \in[1, n]$;

(4) For $u_{i} \in[0,1], i \in[1, n], C\left(\mathrm{u}_{1}, \cdots, u_{i-1}, 0, u_{i+1}, \cdots, u_{n}\right)=0$ all the time;

(5) If the variables $u_{i} \in[0,1], \mathrm{i}=1,2, \cdots, n$ are independent from each other, then $C\left(u_{1}, u_{2}, \cdots, u_{n}\right)=\prod_{i=1}^{n} u_{i}$, noted as $C^{\perp}$; (6)

$$
\text { For any } \quad u_{i}, v_{i} \in[0,1], i=1,2, \cdots, n \text {, }
$$

$\left|C\left(u_{1}, u_{2}, \cdots, u_{n}\right)-C_{2}\left(u_{1}, u_{2}, \cdots, u_{n}\right)\right| \leq \sum_{i=1}^{n}\left|u_{i}-v_{i}\right|$ all the time.

\section{SKLAR THEOREM}

Theorem[8] assumes $\mathrm{H}$ is $\mathrm{n}$-dimensional distribution function, the edge distribution is $F_{1}, F_{2} \cdots, F_{\mathrm{n}}$, then there is an $\mathrm{n}$-dimensional Copula function $\mathrm{C}$, for any $\mathrm{x} \in R^{\mathrm{n}}$.

$$
H\left(\mathrm{x}_{1}, x_{2}, \cdots, x_{n}\right)=C\left(F_{1}\left(x_{1}\right), F_{2}\left(x_{2}\right), \cdots, F_{n}\left(x_{n}\right)\right)
$$

If $F_{1}, F_{2} \cdots, F_{\mathrm{n}}$ is continuous, then $\mathrm{C}$ is unique, conversely, if $\mathrm{C}$ is n-dimensional Copula function, $F_{1}, F_{2} \cdots, F_{\mathrm{n}}$ is distribution function, then the above formula defines an $\mathrm{n}$-dimensional distribution function, its edge distribution is

$$
F_{1}, F_{2} \cdots, F_{\mathrm{n}} \text {. }
$$

From Sklar Theorem, it can be seen that Copula function can independent from marginal distribution of random variables and reflects the correlation structure of random variables, which can divide joint distribution into two separated parts to deal with respectively: the correlations structure among variables and the marginal distribution of variables, in which the correlation structures is described with Copula function.

Inference [9] assumes $X_{1}, X_{2} \cdots, X_{\mathrm{n}}$ are random variables whose distribution function is $F_{\mathrm{x}_{1}}\left(x_{1}\right), F_{x_{2}}\left(x_{2}\right), \cdots, F_{x_{n}}\left(x_{n}\right)$, the joint distribution function is $H\left(\mathrm{x}_{1}, x_{2}, \cdots, x_{n}\right)$, then there is an $\mathrm{n}$-dimensional Copula function that makes (1) set up. If
$F_{\mathrm{x}_{1}}\left(x_{1}\right), F_{x_{2}}\left(x_{2}\right), \cdots, F_{x_{n}}\left(x_{n}\right)$ is continuous, then $\mathrm{C}$ is unique. Otherwise, the uniqueness of $\mathrm{C}$ should be determined by $\operatorname{Ran} F_{1} \times \operatorname{RanF}_{2} \times \cdots \times \operatorname{RanF}_{n}$.

According to Sklar theorem, using Copula function can study the marginal distributions and the correlation structure among variables separately, and it can reduce the difficulty of multivariate probabilistic modeling and analysis.

\section{MECHANICAL SYSTEM RELIABILITY MODEL BASED ON COPULA FUNCTION}

\section{RELIABILITY ANALYSIS OF SERIAL MECHANICAL SYSTEM BASED ON COPULA}

As long as one unit fails, the system will fail, the system is called serial system, which is shown in Figure 1. In mechanical system, the random variables of reliability function often have real physical meaning, such as time, number of cycles, mileage and so on. For ease of presentation, herein it is called life in the analysis.



Fig. 1 The reliability of serial system

Assume the mechanical system is consists of $\mathrm{n}$ units in series, and the life of each unit is $T=\left(T_{1}, T_{2} \cdots, T_{\mathrm{n}}\right)$, the joint distribution function $H\left(t_{1}, t_{2}, \cdots, t_{n}\right)=P\left\{T_{1} \leq t_{1}, T_{2} \leq t_{2}, \cdots, T_{n} \leq t_{n}\right\}, F_{i}(t)$ is the distribution function of $T_{i}$, the reliability function of each component is $R_{i}(t)=1-F_{i}(t)$, the reliability function of system is $R(t)$.

From the Sklar theorem, there is an n-dimensional Copula function $C$ that makes $H\left(t_{1}, t_{2}, \cdots, t_{n}\right)=C^{\mathrm{n}}\left(F_{1}\left(t_{1}\right), F_{2}\left(t_{2}\right), \cdots, F_{n}\left(t_{n}\right)\right)$, in which the $C^{n}(\bullet)$ represents n-dimensional Copula function $\mathrm{C}$, because $F_{\mathrm{i}}(\mathrm{t})$ is continuous, so $C^{\mathrm{n}}\left(F_{1}\left(t_{1}\right), F_{2}\left(t_{2}\right), \cdots, F_{n}\left(t_{n}\right)\right)$ is unique.

When each unit of system is in series, the life of the system is the smallest in all the units, that is $T=\min \left(T_{1}, T_{2} \cdots, T_{\mathrm{n}}\right)$, then the reliability of system can be expressed as:

$$
\begin{aligned}
& R(t)=P\left\{\min \left(T_{1}, T_{2}, \cdots, T_{\mathrm{n}}\right)>t\right\}=P\left(T_{1}>t, T_{2}>t, \cdots, T_{n}>t\right) \\
&= 1-\sum_{i=1}^{n} P\left(T_{\mathrm{i}}-t\right)+\sum_{1 \leq i \leq j \leq n} P\left(T_{i} \leq t, T_{j} \leq t\right)+\ldots+ \\
&(-1)^{k} \times \sum_{1 \leq i_{i} \leq i_{i} \leq \cdots \leq i_{i_{k}}} P\left(T_{i_{1}} \leq t, T_{i_{2}} \leq t, \cdots, T_{i_{k}} \leq t\right)+\ldots+ \\
&(-1)^{n} P\left(T_{i_{1}} \leq t, T_{i_{2}} \leq t, \cdots, T_{i_{k}} \leq t\right)=1-\sum_{i=1}^{n} F_{i}(t)+(-1)^{k} \times \\
& \sum_{1 \leq i_{i} \leq i_{i} \leq \cdots s_{i_{k}} \leq n} C^{n}\left(F_{i_{i}}(t), F_{i_{2}}(t), \cdots, F_{i_{k}}(t), 1,1 \cdots, 1\right), \quad 2 \leq k \leq n
\end{aligned}
$$




\section{RELIABILITY ANALYSIS OF PARALLEL MECHANICAL SYSTEM BASED ON COPULA}

Assume the mechanical system is formed by $\mathrm{n}$ units in parallel, then the life of each unit is $T=\left(T_{1}, T_{2} \cdots, T_{\mathrm{n}}\right)$, $F_{i}(t)$ is the distribution function of $T_{i}$, the joint distribution function is $H\left(t_{1}, t_{2}, \cdots, t_{n}\right)=P\left\{T_{1} \leq t_{1}, T_{2} \leq t_{2}, \cdots, T_{n} \leq t_{n}\right\}$, the reliability function of each component is c, i $=1,2, \cdots, n$ 。

From the Sklar theorem, there is an n-dimensional Copula function $\mathrm{C}$ that makes

$H\left(t_{1}, t_{2}, \cdots, t_{n}\right)=C^{\mathrm{n}}\left(F_{1}\left(t_{1}\right), F_{2}\left(t_{2}\right), \cdots, F_{n}\left(t_{n}\right)\right)$,

When each unit of system is in parallel, the life of the system

is the largest in all the units, that is $T=\max \left(T_{1}, T_{2} \cdots, T_{\mathrm{n}}\right)$, then the reliability of parallel system can be expressed as:

$$
\begin{aligned}
& R(t)=P\left\{\max \left(T_{1}, T_{2}, \cdots, T_{\mathrm{n}}\right)>t\right\}= \\
& =1-P\left(\max \left(T_{1}, T_{2}, \cdots, T_{n}\right) \leq t\right)= \\
& =1-C^{\mathrm{n}}\left(F_{1}(t), F_{2}(t), \cdots, F_{n}(t)\right)= \\
& =1-C^{n}\left(1-R_{1}(t), 1-R_{2}(t), \cdots, 1-R_{n}(t)\right)
\end{aligned}
$$

When each unit is independent, from

$$
\begin{aligned}
& C^{n}\left(1-R_{1}(t), 1-R_{2}(t), \cdots, 1-R_{n}(t)\right)= \\
& =C^{\perp}\left(1-R_{1}(t), 1-R_{2}(t), \cdots, 1-R_{n}(t)\right)= \\
& =\left(1-R_{1}(\mathrm{t})\right)\left(1-R_{2}(t)\right) \cdots\left(1-R_{n}(t)\right)=\prod_{i=1}^{n}\left(1-R_{\mathrm{i}}(t)\right)
\end{aligned}
$$

the $R(t)=\prod_{i=1}^{n}\left(1-R_{\mathrm{i}}(t)\right)$, can be obtained. This is consistent with the parallel system reliability model which is traditional and no considering in the correlation.

So far, this paper has established the mechanical system reliability model based on Copula. So under the premise of not studying the multidimensional random variable joint distribution function, by constructing appropriate Copula function to calculate the reliability value of the mechanical system in the condition of considering the relevance, which can effectively solve the difficult problem of mechanical system reliability modeling.

\section{PARAMETER ESTIMATION}

Copula function parameter estimation methods can be divided into three types: (1) the relevance index method which mainly uses the relationship between parameter $\theta$ of Copula function and Kendall's rank correlation coefficient (as shown in Table 1) to calculate parameter indirect. (2) fitting line method, that is under certain fitting line guideline to calculate the statistical parameter of frequency curve which is optimal to fit with the experience point. (3) maximum likelihood method, for three-dimensional and above Copula function, correlation index method is not applicable clearly, then most of them use the maximum likelihood method to estimate parameter. On the basis of analyzing the first two methods, Xiao $\mathrm{Yi}^{[10]}$ thinks that comparing with the single-variable distribution, the parameter estimation of Copula function has higher requirement for the length of the information. For the middle and small samples, it may result in the bigger estimated value sampling error, and the estimated value is unstable. He uses bootstrap and coupling method to estimate parameter. Statistical test shows that the confidence interval of correlation index method parameter estimated value is

\begin{tabular}{|c|c|}
\hline Name of function & Relationship between $\theta$ and $\mathrm{T}$ \\
\hline $\begin{array}{l}\text { C-H Copula } \\
\text { function }\end{array}$ & $\mathrm{T}=1-1 / \theta$ \\
\hline $\begin{array}{l}\text { Claylon Copula } \\
\text { function }\end{array}$ & $\mathrm{T}=\theta /(2+\theta)$ \\
\hline $\begin{array}{l}\text { AMH Copula } \\
\text { function }\end{array}$ & $\mathrm{T}=(1-2 / 3 \theta)-2 / 3(1-1 / \theta)^{2} \ln (1-\theta)$ \\
\hline $\begin{array}{l}\text { Frank Copula } \\
\text { function }\end{array}$ & $\mathrm{T}=1+\frac{4}{\theta}\left[\frac{1}{\theta} \int_{0}^{\theta} \frac{r}{e^{r}-1} d t-1\right]$ \\
\hline
\end{tabular}
narrow, the result is more stable. The bootstrap can improve the estimation ability of the correlation index method, but the effect is poor for fitting line method. It will result in the severe larger estimated value.

Tab. 1 The relationship between parameter $\theta$ of Copula function and Kendall's rank correlation coefficient $T$

\section{APPLICATION EXAMPLE}

Using mechanical system reliability model based on Copula, by constructing the Copula function which can reflect the relevant structural features of the mechanical system's various componential units, regarding the unit life as a basic input (assuming the life distribution rule of each unit is known), it can estimate the parameter of Copula, thus to expect the reliability of mechanical system. In the following part, take a certain type armored vehicle suspension system for instant to illustrate the application of the mechanical system reliability model based on Copula in the reliability forecast.

\section{DESCRIPTION OF PROBLEM}

The serial mechanical system consists of $\mathrm{n}$ units, the life of i-th unit is $T_{i}$, which distribution function (the 
probability of failure) is recorded as $F_{i}\left(t_{i}\right)$, the reliability is $R_{i}\left(t_{\mathrm{i}}\right)=1-F_{i}\left(t_{i}\right)$, a set of observation value of each unit is known $\left(t_{1 j}, t_{2 j}, \cdots, t_{n j}\right), \mathrm{j}=1,2, \ldots, \mathrm{w}$, try to estimate the system reliability.

By the formula (2), it can be obtained that:

$$
\begin{aligned}
& \mathrm{R}(\mathrm{t})=1-\sum_{i=1}^{n} F_{i}(t)+(-1)^{k} \times \\
& \sum_{1 \leq i_{1} \leq i_{2} \leq \cdots \leq i_{k} \leq n} C\left(F_{i_{1}}(t), F_{i_{2}}(t), \cdots, F_{i_{k}}(t)\right)=1-\sum_{i=1}^{n} F_{i}(t)+(-1)^{k} \times \\
& \quad \sum_{1 \leq i_{1} \leq i_{2} \leq \cdots \leq i_{k} \leq n} C^{n}\left(F_{i_{1}}(t), F_{i_{2}}(t), \cdots, F_{i_{k}}(t), 1,1 \cdots, 1\right), 2 \leq k \leq n
\end{aligned}
$$

In the above formula, $F_{i}(t)$ can be obtained by determining the distribution type and its distribution parameter. First, select the appropriate Copula model, by the nature of Copula function, estimate the parameter

of $\sum C^{\mathrm{n}}\left(F_{1}(t), F_{2}(t), \cdots, F_{n}(t)\right) \quad$ to make $\quad F_{j}(t)=1$ $j=i_{k}+1, i_{k}+2, \cdots, n$, so you can get $1 \leq i_{1} \leq i_{2} \leq \cdots \leq i_{n}$, $2 \leq k \leq n$.

Thus, the basic steps of using the mechanical system model based on Copula to expect the system reliability:

1. Determine the marginal distribution, that is the distribution type $F_{i}(t)$ of unit life, and estimate the distribution parameter;

2. Choose an appropriate Copula function, to make it can describe the relevant characteristics of each unit life well;

3. Estimate the parameter of Copula model;

4. Calculate system reliability.

\section{CONSTRUCTING COPULA MODEL}

In view of the correlation among the mechanical parts life usually shows the positive correlation, and at the same time, taking into account of the simple requirement to the model parameter estimation and calculation, this paper chooses the multi Gumbel Copula function in Archimedean Copula function family. Gumbel Copula function can accurately portray the correlation of mechanical system. The distribution function and density function of multi Gumbel Copula function respectively is:

$$
\begin{gathered}
C\left(\mathrm{u}_{1}, u_{2}, \cdots, u_{n} ; \theta\right)=\exp \left(-\left[\sum_{i=1}^{n}\left(-\ln u_{i}\right)^{1 / \theta}\right]^{\theta}\right), \\
c(u, v ; \theta)=\frac{\partial C\left(u_{1}, u_{2}, \cdots, u_{n} ; \theta\right)}{\partial u_{1} \partial u_{2} \cdots \partial u_{n}}
\end{gathered}
$$

In the formula, $\theta \in(0,1]$ is the correlation coefficient, $\theta=1$ represents the random variables $u_{1}, u_{2}, \cdots, u_{n}$, is independent, $\theta \rightarrow 0$ represents the random variables $u_{1}, u_{2}, \cdots, u_{n}$ tend to full correlation.

$$
\begin{aligned}
& \text { If } u_{i}=F_{i}\left(t_{i}\right)=1-e^{-\left(t_{i}-\gamma_{i}\right) m_{i} / \eta_{i}^{m}} \text {, from formula (6), it can get } \\
& \qquad \begin{array}{l}
C^{\mathrm{n}}\left(F_{1}\left(t_{1}\right), F_{2}\left(t_{2}\right), \cdots, F_{n}\left(t_{n}\right) ; \theta\right)= \\
=\exp \left(-\left[\sum_{i=1}^{n}\left(-\ln F_{i}\left(t_{i}\right)\right)\right]^{1 / \theta}\right)
\end{array}
\end{aligned}
$$

Then, according to formula (5), the system reliability is

$$
\begin{gathered}
\mathrm{R}(\mathrm{t})=1-\sum_{i=1}^{n} F_{i}(t)+(-1)^{k} \times \\
\sum \exp \left(-\left[\left(-\ln \mathrm{F}_{\mathrm{i} 1}(t)\right)^{1 / \theta}+\left(-\ln F_{i 2}(t)^{1 / \theta}\right)+\cdots+\left(-\ln F_{i n}(t)\right)^{1 / \theta}\right]^{\theta}\right)
\end{gathered}
$$

It needs to estimate the value $\hat{\theta}$ of Copula function's parameter $\theta$, then make $F_{j}(t)=1, j=i_{k}+1, i_{k}+2, \cdots, n$ Put $\hat{\theta}$ and the Weibull distribution parameter estimated value $\hat{\gamma}_{i}, \hat{m}_{i}, \hat{\eta}_{i}$ of each unit into (9), the system reliability can be obtained.

\section{ESTIMATE SYSTEM RELIABILITY}

From 3.2 to calculate the system reliability function, if estimating the reliability of the suspension system when the vehicle is traveling to $6000 \mathrm{~km}$, put the $\mathrm{t}=6000 \mathrm{~km}$ into reliability function equation, the $(6000)=0.532$ can be obtain.

Without considering the correlation among the units, the reliability of suspension system when the vehicle is traveling to $6000 \mathrm{~km}$ is $\mathrm{R}$ ' $(\mathrm{t})=0.425$. Hydraulic shock absorber is the reliability lowest unit in the suspension system. When the vehicle is traveling to $6000 \mathrm{~km}$, the reliability is $R_{3}(6000)$ $=0.535$.

If the system reliability based on Copula function is $R_{\mathrm{c}}(t)$, assuming the system reliability when the units are independent from each other is $R_{I}(t)$ the corresponding system reliability of the weak link theory is $R_{W}(t)$. From the calculation results of the above examples, $R_{\mathrm{c}}(t)=0.532$, $R_{I}(t)=0.425, R_{W}(t)=0.535$, Then clearly there is $R_{I}(t) \leq$ $R_{\mathrm{c}}(t) \leq R_{W}(t)$.

This is consistent with the conclusion of reliability margin model, which also verifies the reasonableness of mechanical system reliability model based on Copula function.

The many years' actual use data of the armed forces show that this equipped suspension system' travelling damage rate of $6000 \mathrm{~km}$ is $48 \%$, which is equivalent to the suspension system' reliability is 0.520 when using it to $6000 \mathrm{~km}$, slightly 
lower than the expected value 0.532 . Excluding the damage caused by human operation error and improper repairs, it can be considered that the model is basically consistent with the actual situation. This confirms the correctness and rationality of the model from the theory and practice aspects.

\section{CONCLUSIONS}

Using Copula theory to carry out mechanical system reliability modeling and its expectation to study the distribution of random variables (marginal distribution) of the mechanical product life and the associated structure of variables separately, which reduces the difficulties of multivariate probabilistic modeling and analysis, and make the modeling and analysis process more clearly. Based on the theory make a detailed analysis to the serial and parallel system, proposes a method to calculate the system reliability of the components related serial system. The method takes into account the correlation of the various components in the system to make the results of system reliability more reasonable, which provides a new method for evaluating the reliability of the suspension system. The correlation problem of mechanical system reliability is widespread and unavoidable. The proposal of the mechanical system reliability model based on Copula function provides a scientific and practical approach for solving the problems of mechanical system reliability modeling and expectation.

\section{BIBLIOGRAPHY}

1. WEI Yanhua, ZHANG Shiying. Copula Theory and Its Applications in Financial Analysis [M]. Beijing: Tsinghua University Press, 2008: 1-33.

2. ZHANG Yaoting. Copula Technology and Financial Risk Analysis [J]. Statistical Research, 2002, (4):48-51.

3. LUO Junpeng. Copula Theory and Its Application Study on Financial Analysis [J]. Tianjin: Tianjin University, 2005.

4. Zieja M.; Wazny, M. A model for service life control of selected device systems [J]. Polish Maritime Research, 2014, 21(2) 45-49.

5. TANG Jiayin, ZHAO Yongxiang, HE Pingdeng. Copulas New Theory for Reliability Calculation Involving Correlation in Mechanical Systems [J]. Mechanical Science and Technology, 2009, 28(4): 532-535.

6. HOU Bin. Application of Copulas in reliability theory [J]. Journal of Southwest University for Nationalities: Natrual Science Edition, 2008, 34(4):660-668.

7. H Joe. Multivariate Models and Dependence Concepts [M]. Chapman \& Hall, 1977.

8. Sklar A. Fonctions de repartition an dimensions et leura marges [J]. Publication de I' insititut de Statictique de I'
Universite de Paris, 1959, 8:229-231.

9. Nelson R B. An introduction to Copulas[M]. Springer, New York, 1999.

10. Atkins, R J; Tidd, M; Ruffo, G.[J]. Sturgeon Bank, Fraser River Delta, BC, Canada: 150 Years of Human Influences on Salt Marsh Sedimentation [J]. Journal of Coastal Research, 2016, SI 75, 790-794.

11. YI Wende. Reliability of the dependent components in simple system [J]. Journal of Western Chongqing University (Nature Sciences Edition), 2005, 4(2): 5-8.

12. XIAO Yi. Study on Multivariate Hydrological Analysis Calculation Based on Copula Function. [D]. Wuhan: Wuhan University, 2007.

\section{CONTACT WITH THE AUTHOR}

Hai AN

School of Aerospace and Civil Engineering Harbin Engineering University Harbin 150001 Heilongjiang CHINA 